\title{
Technologies et techniques des sports: le regard de l'histoire et des sciences humaines et sociales
}

\author{
Luc Robène* \\ Université de Bordeaux, UMR Thalim 7152, Bordeaux, France
}

Reçu le 8 novembre 2017, Accepté le 21 novembre 2017

\section{La définition d'un périmètre}

La technologie désigne l'étude des outils et des techniques. Entendu dans son acception première, comme discours sur la technique (Ellul, 1988), le terme recouvre l'ensemble des discours qui définissent, dans le temps, l'état de l'art d'un secteur de l'activité humaine en matière d'outils et de savoir-faire incluant les arts, l'artisanat, les métiers, les sciences appliquées voire éventuellement les connaissances. Par extension, la technologie fait aujourd'hui référence aux systèmes et aux méthodes d'organisation qui rendent possible un niveau particulier d'activité et de conception (potentiel technologique) ainsi que tous les domaines d'étude et les produits qui en résultent.

L'articulation entre les techniques (attachées aux 《arts») et les sciences, ainsi que la connaissance et l'optimisation des conditions de cette convergence, constituent le cœur de ce qui va définir, progressivement, la conception moderne du terme «technologie».

À la fin $\mathrm{du} \mathrm{xVIII}^{\mathrm{e}}$ siècle, cette articulation procède d'abord de manière concrète autour de quelques moments remarquables. La conquête de l'air naissante, par exemple, et l'invention du «plus léger que l'air » (aérostats : ballons gonflés à l'hydrogène et montgolfières gonflés à l'air chaud-1783), en s'adossant pour partie à l'essor de la chimie pneumatique, créent en retour un horizon d'attente technique favorable au développement des sciences fondamentales (Robène, 1998). Dans le contexte de la révolution scientifique et des démonstrations spectaculaires, la fabrication de «l'air inflammable » (hydrogène) en très grande quantité rendue nécessaire pour gonfler les aérostats (ballons à hydrogène inventé par le physicien Jacques Charles), stimule les tentatives de perfectionnement des méthodes de production. Cette dimension pratique donne aux savants comme Lavoisier l'occasion de réaliser, dans un contexte porteur, des expériences essentielles pour la chimie moderne à l'instar de la double expérience cruciale de décomposition et recomposition de l'eau, en février-mars 1785 (Bret, 2004). Par ailleurs, l'expérimentation des propriétés physiques de l'air chaud

\footnotetext{
*Auteur correspondant: luc.robene@u-bordeaux.fr
}

qui a rendu possible, empiriquement, les premiers envols de montgolfières et la réalisation du premier vol humain, met les théoriciens et savants face à de nouveaux défis mathématiques (Euler) et physiques (Saussure).

Dans le contexte de la révolution scientifique, puis de la révolution industrielle naissante, l'un des enjeux de la technologie en tant que prisme de connaissance devient dès lors de définir les conditions plus précises d'une fusion entre les sciences et les «arts utiles». Cette articulation qui fonde au $\mathrm{xIX}^{\mathrm{e}}$ siècle la conception de la technology anglo-américaine donne leur vocation à des institutions prestigieuses de la Nouvelle Angleterre comme le MIT de Boston (Massachussetts Institut of Technology).

Certes, l'essor de cette configuration technologique entendue comme synergie ou comme alliage est également imputable aux philosophies progressistes des Lumières qui dès la fin du XVIII ${ }^{\mathrm{e}}$ siècle tendent à considérer l'homme dans sa perfectibilité c'est-à-dire très concrètement dans ses capacités à améliorer les conditions de son existence par ses propres moyens. Les techniques, les sciences, l'éducation, la raison deviennent ensemble les instruments d'une transformation du monde au terme de laquelle, échappant à la vision aristotélicienne qui le contraint, l'homme se rend maître de la nature et donc maître de sa propre destinée. La maîtrise d'un ensemble de connaissances et de pratiques permet donc d'agir dans le sens d'un progrès idéalisé. La performance humaine en tant que telle peut désormais être pensée... C'est aussi dans la définition de ce paradigme du progrès indéfini (Condorcet, Turgot) que l'activité du corps fonde sa légitimité en tant que domaine d'éducation et bientôt comme levier de performance. D'une part, des systèmes de gymnastique alliant connaissances scientifiques (médicales notamment) et pratiques (techniques et répertoires gestuels) sont formalisés par des théoriciens, pédagogues et médecins comme autant d'outils de redressement des corps (l'orthopédie chez Nicolas Andry, 1741) et comme moyens et supports pédagogiques englobés plus généralement dans des philosophies de l'éducation, comme celle de Rousseau (Emile ou de l'éducation, 1762). D'autre part, l'appréhension des gestes techniques s'agrège ou s'intègre à des systèmes de connaissances encyclopédiques dont on 
recense de manière plus systématique la portée et les périmètres dans le domaine des «arts » (Robène, 2008).

D'une certaine manière, on trouve chez Leibnitz les bases de cette réflexion. Le philosophe, annonçant au XVII ${ }^{\mathrm{e}}$ siècle le travail des encyclopédistes, avait en effet déjà entrevu la valeur des gestes techniques et l'intérêt de leur prise en considération au-delà de la simple matérialisation des objets qu'ils rendaient possibles : «Il n'y a point d'art mécanique si petit et si méprisable, qui ne puisse fournir quelques observations ou considérations remarquables et toutes les professions ou vocations ont certaines adresses ingénieuses dont il n'est pas aisé de s'aviser et qui néanmoins peuvent servir à des conséquences bien plus élevées » (Leibnitz, 1890).

Cette approche par les utilités raisonnées des gestes techniques, des tours de main et des habiletés du corps, bientôt par les «performances du corps », va bénéficier au $\mathrm{xIX}^{\mathrm{e}}$ siècle de prolongements rationnels inscrits dans le développement conjoint des sciences et des techniques au sein de dispositifs innovants. D'une part, la technologie relative aux usages et à l'éducation du corps bénéficie des apports de la science, adossée à des inventions techniques remarquables. Marey par exemple, qui analyse par la chronophotographie le vol des oiseaux (Robène, 1998) et qui bientôt décortique les attitudes, postures et gestes humains, assisté de Demeny, est à l'origine de conceptions nouvelles de l'éducation physique (Pociello, 1999). Marey génère également par ses analyses une approche tout à fait neuve du mouvement et de la mobilité (il est accessoirement à cette occasion, avec Demeny, l'un des co-inventeurs du cinéma) dont le monde du sport et de l'éducation physique se servira longtemps, réifiant le geste du champion étudié au prisme des kinogrammes: ces schémas analytiques issus d'une technologie d'imagerie moderne ont été longtemps publiés en France dans le revue EPS. D'autre part, dans le prolongement des conceptions attachées à l'idéologie du progrès, le culte de la performance dont le sport moderne constitue désormais l'épure, devient l'horizon du monde moderne, un «modèle global » pour une société de performances (Ehrenberg, 1991). Activité technicisée et technologisée par excellence, le sport constitue désormais la grille de lecture et de référence des sociétés modernes, des sociétés «compétitives», confrontées implicitement à l'idéologie technicienne qu'elles ont forgée (Habermas, 1973 ; Robène \& Bodin, 2014)

C'est dans cette perspective historique qu'il convient de repenser l'alliage des techniques et des technologies du sport ainsi que les enjeux attachés à la performance et aux formes d' «empowerment » et d' «enhancement» qui lui sont associées ${ }^{1}$.

\footnotetext{
${ }^{1}$ Empowerment signifie littéralement: octroi du pouvoir. La notion par extension renvoie aux conditions qui définissent l'accroissement de l'autonomie des individus et des groupes face aux situations économiques, sociales, politiques, écologiques auxquelles ils sont confrontés. Enhancement signifie littéralement renforcement ou augmentation. C'est l'utilisation de moyens technologiques pour sélectionner ou modifier les caractéristiques et les capacités humaines, que l'altération se traduise ou non par des caractéristiques et des capacités qui se situent au-delà de la portée humaine existante.
}

\section{Retour sur l'histoire des techniques du sport}

Il est difficile en effet lorsqu'on évoque l'histoire du sport de ne pas s'attacher à la dimension technique et par extension aux périmètres technologiques de l'activité. Tout dans le sport moderne, en effet, renvoie à la technique et aux technologies (Robène \& Léziart, 2006): des conditions de la performance (efficacité du geste, formes d'entraînement, optimisation du matériel et trouvailles, évolution des espaces et du règlement, etc.), à l'invention des modèles et des stratégies de jeu, jusqu'au coaching, en passant par les développements des technologies qui entretiennent une connivence certaine avec le paradigme $\mathrm{du}$ record (analyse du geste et écriture du mouvement, imagerie, numérisation, technologie des matériaux, des vêtements, des instruments et des machines, des moyens de communication et des médias, voire des procédés d'amélioration de la performance comme le dopage).

Comme nous le soulignons supra, le problème mérite cependant d'être repensé en fonction d'un cheminement. Car l'idée même de «technique »-ainsi que par extension l'idée de «technologies» et de savoirs inscrits dans la genèse de liens étroits entre théorie et pratique - appliquée au contexte sportif ne va pas de soi.

Dans la Grèce antique, la technè (technique) renvoie à l'idée de fabrication, de production matérielle, avant de signifier, par extension, la méthode, la manière, la façon ou le faire efficace (Castoriadis, 1995). Longtemps opposée au logos (le parler-penser) par les modernes et généralement sous-estimée, la technique est aujourd'hui reconnue comme l'une des grandes composantes du savoir-faire humain. L'homme invente et transmet en permanence des moyens de rendre la vie plus facile, plus agréable, plus efficace. À l'instar du langage, la technique permet à l'humanité de s'extraire d'une temporalité assujettie à l'ordre naturel : elle permet un dégagement de l'immédiat (Leroi-Gouhran, 1964). Les techniques corporelles, qui correspondent aux moyens physiques transmissibles jugés les plus adéquats pour atteindre un but (le geste efficace), intègrent ce processus libératoire. Dans cette perspective, l'invention des techniques sportives peut être appréhendée comme «un moment dans une histoire des efficiences et des opérationalités physiques » (Vigarello, 1988). L'intelligence humaine compense dans le cadre particulier d'une pratique culturelle à forte valence symbolique, la faiblesse des hommes ou leurs limites en puissance musculaire et leurs difficultés à maîtriser l'environnement physique. L'inventivité humaine se manifeste dans la transformation des techniques et des outils techniques, supports des activités physiques et sportives. Le rapport à la technique constitue dès lors un analyseur pertinent pour comprendre le sens que les pratiques acquièrent au fil du temps. Cet aspect essentiel éclaire par exemple la distinction désormais classique entre sport antique et sport moderne. La gymnastique des Grecs correspond à une forme de finitude dans laquelle la technique corporelle n'a pas pour horizon le dépassement de soi au sens de la performance, mais bien l'exécution parfaite d'une prestation qui définit et qualifie 
le sujet par ses actes et la moralité de ses actes. Le sport moderne, qui s'affirme dans l'Europe industrielle du XIx ${ }^{\mathrm{e}}$ siècle, trouve au contraire ses significations et sa symbolique dans l'idée de perfectibilité humaine, héritage des Lumières traversé par le concept de «progrès indéfini » (Condorcet, Turgot). La technique sportive devient le vecteur d'une efficacité corporelle inédite, ouverte sur la production de performances et de records, traversée par de nouvelles préoccupations liées au rendement (entraînement).

$\mathrm{Au}$ plan anthropologique, cette modernité sportive suggère cependant quelques questions fondamentales. Ainsi, dès 1934, Marcel Mauss conceptualise la notion de «technique du corps » en montrant combien le geste technique est une construction éminemment culturelle (Mauss, 1950). Pourtant Mauss tout en s'appuyant sur quelques exemples «sportifs» (nage, course) peine à intégrer dans sa classification les techniques sportives. D'une part, ces techniques ne sont pas des techniques traditionnelles. D'autre part, elles ne se distribuent pas en fonction de l'âge et du sexe. Elles échappent donc aux principes essentiels de la classification maussienne. Plus largement, l'idée de techniques corporelles que Mauss définit comme les manières par lesquelles les hommes, société par société, d'une façon traditionnelle, savent se servir de leur corps, s'oppose à la progressive diffusion et universalisation des techniques sportives au $\mathrm{xx}^{\mathrm{e}}$ siècle. Enfin, l'efficacité de la technique corporelle traditionnelle, inscrite dans l'ordinaire des jours (dormir, manger, s'asseoir, chasser, etc.), se distingue de l'efficience des techniques du sport moderne dont la raison s'est construite au regard de motricités placées artificiellement sous contraintes (compétition et règlements, normalisation des conditions de la performance, déréalisation des obstacles et standardisation des espaces, etc.)

En définitive la place de la technique dans le sport doit être pensée dans le rapport nouveau que l'efficacité gestuelle instaure à partir de la fin du $\mathrm{XIX}^{\mathrm{e}}$ siècle avec des buts de maîtrise et de compétition en apparence détachés de l'immédiateté et des contraintes de la vie sociale: passage d'une efficacité quotidienne réglée sur l'utilité des gestes (se sauver, faire la guerre, monter à cheval, nager) à l'efficience sportive; glissement de «l'art de...», souvent propriété sociale d'une élite ou d'un corps professionnel (les arts libéraux, les arts académiques) à la production d'un geste expert inscrit dans l'univers artificialisé des situations sportives.

Plusieurs problématiques permettent alors d'éclairer cette histoire. Vigarello (1988) montre ainsi comment les transformations des techniques sportives s'organisent autour de variations plus ou moins brutales dans l'ordre des motricités, des schèmes d'action et dans l'utilisation des différentes surfaces et segments du corps: loi d'intégration, variations restreintes, ruptures mais également transpositions de modèles. Cette archéologie du geste sportif, dévoilée par l'analyse minutieuse des essais, des tâtonnements, permet de rendre compte des formes de généalogies qui organisent l'espace de production des gestes sportifs. La technique du crawl, par exemple, trouve son origine dans les variations successives, à la fin du XIX ${ }^{\mathrm{e}}$ siècle, de plusieurs modèles techniques issus de la brasse: brasse traditionnelle, «indienne», over arm side stroke, puis trudgen. L'analyse des évolutions de la technique du lancer de poids permet par ailleurs de saisir comment, phase après phase, sont mobilisés de nouveaux segments corporels (bras, tronc, jambes) et réagencées les actions de l'athlète (allongement du chemin de lancement, rotation du corps et utilisation de la force centrifuge). La transposition de modèles constitue une autre voie explicative qui permet de montrer comment certaines solutions motrices construites dans un domaine trouvent à s'appliquer dans d'autres. La volte, initialement conçue pour lancer le disque, s'est évadée du côté du lancer de javelot avec un succès tel que devant les performances jugées dangereuses pour le public le règlement de 1956 a interdit la nouvelle technique.

Ce dernier point est fondamental car il souligne l'importance de l'interaction règlement-techniques. Il montre combien est décisive dans le processus d'invention des techniques sportives l'interdépendance entre la logique interne d'une activité, ses règles, et les conditions de réalisation (matérielles, symboliques, culturelles) dans lesquelles s'insère l'innovation technique. L'élimination progressive des techniques les plus violentes comme le hacking (coups de pied portés sur un joueur) en rugby accentue cet effet en rappelant comment l'espace du jeu ou de la compétition intègre des choix qui contraignent l'activité technique du sportif en la réinsérant dans un ensemble beaucoup plus vaste de déterminants.

L'histoire des techniques sportives peut alors être lue au prisme de deux approches complémentaires: d'une part, l'histoire technique de la technique (Fèbvre, 1935), qui privilégie la dynamique interne du jeu, de la spécialité sportive et de ses contraintes matérielles et réglementaires; d'autre part, l'histoire englobante qui réinscrit cette évolution dans un ensemble plus large de changements: représentations et sensibilités, modèles culturels, sociaux, repères esthétiques, évolutions technologiques, dynamiques économiques, etc. L'invention des positions de recherche de vitesse, en ski, dans les années 1960, se situe bien à l'articulation de transformations qui intègrent à la fois des variations techniques propres à la discipline sportive (positions du corps, attitudes, matériel), de nouvelles sensations (glisse) et des données issues des études sur l'aérodynamisme provenant de l'ensemble des secteurs d'activité industriels liés à la mobilité (automobile, aéronautique, etc.). Les archives montrent très concrètement de quelle manière les firmes aéronautiques comme la maison Farman par exemple ont largement contribué dans l'Entre-deux-guerres au développement des skis nautiques modernes (Fig. 1).

\section{Une idéologie de l'empowerment et de l'enhancement?}

Une perspective importante du travail à entreprendre consiste donc à éclairer le «redoublement technique de la technique» (Robène \& Léziart, 2006). C'est-à-dire les 


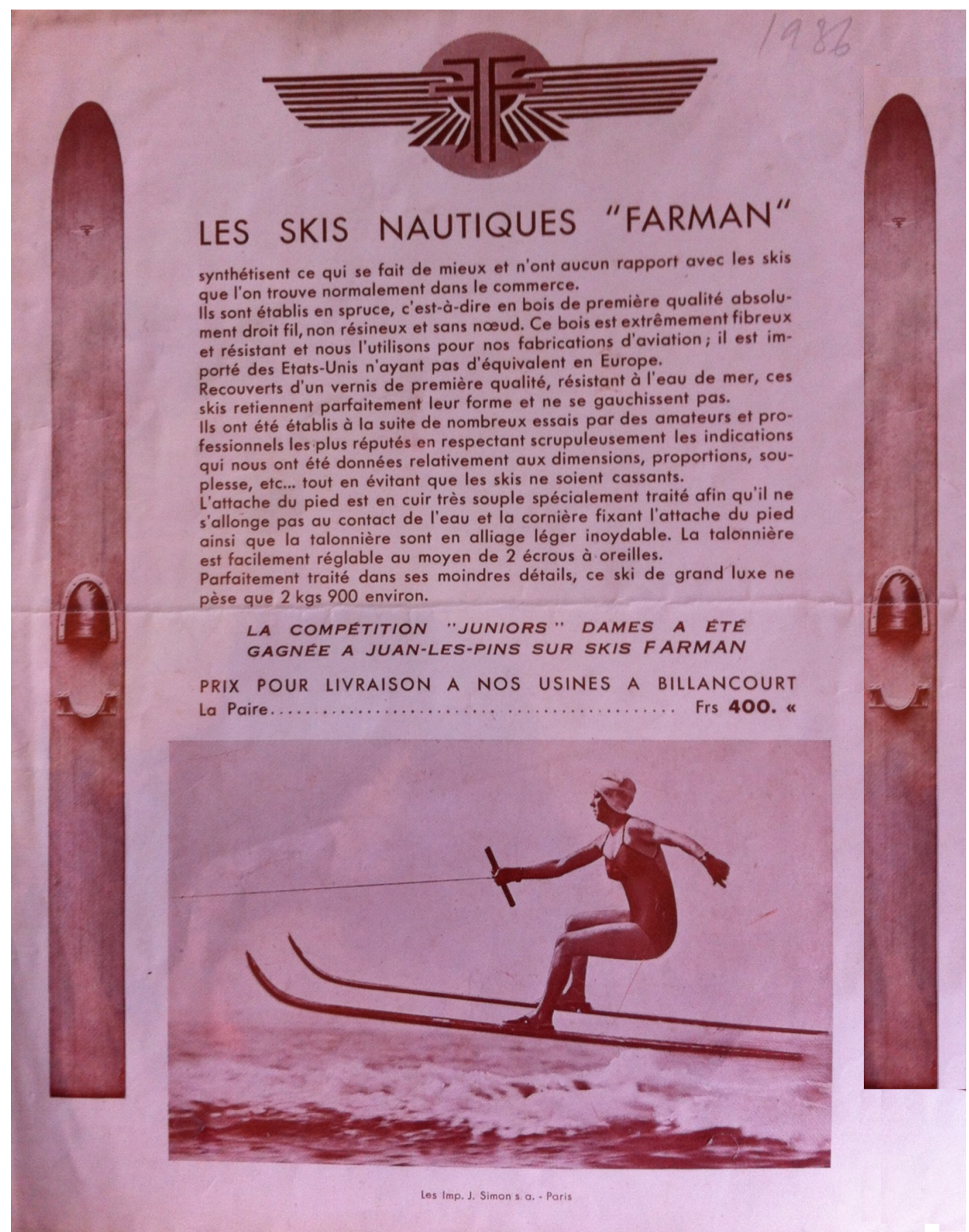

Fig. 1. Publicité de la firme aéronautique Farman pour la fabrication de skis nautiques (1936).

Transferts technologiques de l'aviation vers le sport ». (Collection auteur).

rapports qui s'établissent entre d'une part l'activité technique du sujet et d'autre part le travail scientifique et technique -i.e. technologique - qui vient soutenir le développement et l'affinement des techniques sportives. Ces aspects concernent aussi bien les explorations de l'individu par la maîtrise des techniques et technologies modernes (techniques d'investigation de l'état de forme des sportifs, de leur récupération, de leur amélioration corporelle) que les problèmes liés à l'utilisation de nouvelles techniques d'analyse du mouvement ou de l'amélioration des techniques d'information et de communication, de l'informatique et des logiciels supposés se placer au service du sport et de la performance. D’une manière générale, l'évolution du «milieu technique » qui intègre l'innovation technologique est décisive dans la transformation des gestes. L'adoption de nouveaux matériaux comme la fibre de verre a transformé les techniques du saut à la perche; l'utilisation de mousses synthétiques dans les aires de réception a suggéré de nouvelles audaces techniques (figures acrobatiques en gymnastique, réception sur le 
dos en saut en hauteur). L'étude des modèles d'instruments constitue également une piste intéressante. Comme le montre Joël de Rosnay dans sa typologie des engins de glisse issus du surf, l'hérédité et la filiation entre les machines (du surf au kite surf en passant par le wind-surf) participent à générer des filiations et des formes de transposition au niveau des techniques sportives.

L'aérodynamique a permis de construire des voitures de sport au design particulier, permettant ainsi de réduire les turbulences du vent contre la voiture, de diminuer leur consommation et même d'utiliser la force du vent pour plaquer la voiture contre le sol. L'hydrodynamique a non seulement permis de confectionner des vêtements aquatiques qui glissent plus facilement dans l'eau, réduisant ainsi l'action de l'eau contre le corps, mais aussi de perfectionner chaque type de nage. Les ballons ne sont plus des organes comme dans le passé (il était fréquent d'utiliser des vessies, des panses de brebis ou d'autres animaux pour jouer au ballon), les raquettes, les roues des cyclistes ne sont plus «en boyaux» mais mobilisent une technologie et une physique des matériaux absolument nouvelles. Les chaussures de sport que nous utilisons aujourd'hui nous permettent d'amortir les chocs, de protéger nos chevilles, nous préservant ainsi de blessures. Les vêtements techniques ont envahi la sphère sportive. Bref la technologie, production humaine, «agit» sur le sport et le transforme, définissant constamment de nouveaux horizons de performance.

En 1974, avec un ratio de 95,7\% de matchs gagnés, le tennisman américain Jimmy Connors devenait numéro 1 mondial en multipliant les records, aidé par la première raquette en acier à tête ronde, la Wilson T2000. En 1993, le cycliste amateur écossais Graeme Obree battait le record du monde de l'heure cycliste grâce à un vélo bricolé avec, entre autres, des pièces de sa machine à laver. Aujourd'hui, la goal line permet un arbitrage plus précis et est adoptée par les championnats de football français, allemand et italien, afin de s'assurer que le ballon a bien franchi la ligne des buts.

Pour les Jeux Olympiques 2024, le patch électronique fera son apparition afin de mesurer la fréquence cardiaque des athlètes cherchant à battre des records d'endurance. Actuellement, l'interaction sport-numérique devient un horizon proche : le numérique au service de la performance, le centre média, le stade connecté, le sport virtuel et l'eparcours forme physique ne sont plus des fantasmes technologiques mais bien des lignes qui tracent le futur du sport. On imagine transformer tout appareil de la vie quotidienne en objet connecté et des firmes dessinent le laboratoire portatif du sportif de demain: un cardiofréquencemètre de poitrine pour générer le rythme cardiaque et la géolocalisation de l'athlète.

Certes, il existe aujourd'hui des limites réglementaires aux dispositifs qu'un athlète peut porter sur lui durant les compétitions, mais il est probable qu'elles seront modifiées. Un système de contrôle des conditions physiques - fréquence cardiaque, température, hydratation, glycémie, fatigue musculaire, etc. - associé à un localisateur GPS permettrait de prévenir de graves accidents, en particulier dans des sports comme le marathon, le triathlon, le ski de fond ou la natation en eau libre, où l'engagement physique est extrême et l'environnement parfois hostile. Un signal avertirait l'athlète de la détérioration des paramètres de suivi, et une alarme indiquant la position du concurrent serait envoyée simultanément au médecin de la compétition en vue d'intervenir le plus rapidement possible. Le souci de sécurité s'imposera tôt ou tard et deviendra même probablement obligatoire, comme l'est aujourd'hui la bombe pour les cavaliers ou le casque pour les boxeurs amateurs.

Grâce à la miniaturisation de l'électronique, le laboratoire d'analyses portable de l'athlète tiendra dans un badge ou une montre. L'entraîneur pourra dès lors accéder en temps réel à ces données, afin de gérer de façon optimale les ravitaillements pendant les épreuves les plus longues, à l'image du Tour de France. L'athlète absorbera des nutriments seulement lorsque son organisme en aura besoin, tout comme les voitures de F1 ne s'arrêtent pour changer de pneus que lorsqu'un capteur indique aux techniciens que la gomme a perdu son adhérence ou a trop chauffé.

Finalement, les techniques et technologies sportives sont historiquement signifiantes car elles constituent, à leur manière, des moments datés et situés dans une démarche d'adaptation motrice ou de résolution de problèmes posés par un ensemble de contraintes liées à la variété des milieux et des espaces considérés, à la diversité des situations codifiées, des différentes configurations possibles de relations entre les acteurs, des règles imposées et de leurs changements ou de leurs contournements, des imaginaires et des normes qui trament l'épaisseur et le sens donné aux activités physiques et sportives, des matériaux disponibles, des engins utilisés et des supports scientifiques et techniques qui croisent et secondent l'activité technique à proprement parler. Reste à comprendre dans cet ensemble complexe les limites qui légitiment l'invention du geste ou de l'appareillage techniques dans un cadre éthique et réglementaire singulier, orienté par la notion d'équité. Les performances de l'athlète handicapé Oscar Pistorius, équipé de prothèses en carbone (jambes artificielles) et capable de concurrencer voire de surpasser les athlètes valides, sont venues dans une période récente questionner de manière originale la place de la technique et des technologies dans le sport. L'épisode des combinaisons de natation a également permis de pointer des problèmes liés aux conditions de réalisation de la performance: suite à un trop grand nombre de records battus depuis leur apparition dans la natation, les combinaisons $100 \%$ polyuréthane sont finalement interdites, accusées de prendre le pas sur l'exploit physique. L'histoire des techniques et des technologies devrait également questionner la place de tout dispositif technique y compris ceux que le sport a du mal à réguler, comme le dopage. Une manière sans doute de réinterroger à travers l'invention des techniques sportives et des technologies, sans cesse reconduite, le sens profond du sport. Car le domaine des transformations 
semble infini. Et c'est bien ce qui constitue le cadre problématique des liens entre sport et technologies.

Dans son article Technology in sport: Three idealtypical views and their implications, Sigmund Loland définit la technologie comme "humans-made means to reach human interests and goals» (Loland, 2002: 2-3). Nous touchons ici à un aspect fondamental. En effet, la technologie est définie non pas seulement par ce qu'elle «est», mais bien par ses effets. Ce que nous attendons de la technologie est qu'elle affecte positivement nos vies et nos manières d'agir, qu'elle optimise nos capacités, nos pouvoirs d'agir sur le monde, qu'elle donne lieu à une production tangible. La technologie est fondamentalement liée à un projet qui guide son élaboration. La définition de Loland (2002) souligne le fait que la technologie est une production humaine et donc, révèle une forme d'artificialité (face à une naturalité idéalisée). Loland soulève donc simultanément une autre problématique : celle du lien complexe entre le «naturel» ou plutôt la «nature » de l'Homme et le «technologique». Et c'est ici l'épineuse question de l'augmentation de l'humain (le fameux human enhancement anglo-américain) qui se dessine. Quel sens donner à la technologie dans son rapport à l'Homme? Le paléoanthropologue Coppens (2009) émet l'idée que la «technologisation » de l'homme débute avec la maitrise du feu par Homo erectus. Dans cette perspective anthropologique, ne doit-on pas considérer la technologie comme l'essence même de l'Humain?

\section{Sports et technologies: de nouveaux questionnements}

C'est dans cette perspective que ce numéro spécial «Technologies et techniques des sports» prend tout son sens. Car de l'histoire des techniques à l'histoire de l'innovation, la construction sociale des technologies du sport ouvre un champ de questions et de recherches prometteur (Robène, 2014). Dans la perspective de la technologie culturelle et des technology studies, sans pour autant s'y réduire, l'approche interdisciplinaire permet de repenser ce problème et d'envisager des thématiques nouvelles inscrites dans l'approche des réseaux techniques et des macro-systèmes (économie, science, géographie, monde du travail, politique, sciences du vivant, etc.), à l'articulation du façonnement technologique du social et du façonnement social des techniques.

L'objectif de ce numéro spécial consiste donc à questionner de manière interdisciplinaire les rapports pluriels et interactifs qu'entretiennent les pratiques sportives et la technologie, non comme des espaces aux projets disjoints, contraints par des oppositions ou des clivages, mais bien comme des arènes de créativité et d'excellence à connections technologiques multiples dont il convient de scruter le modelage et les évolutions au regard des enjeux culturels, sociaux, économiques, qui définissent le périmètre du sport contemporain.

L'article de Perera, Villoing, Ruffié et Gosset, comme celui de Howe et Silva, questionne la technologie mobilisée dans les pratiques sportives à destination des personnes handicapées. Le premier porte sur l'expérience du Fauteuil Tout Terrain, technologie rendant accessible la montagne aux personnes paraplégiques ou tétraplégiques. À partir d'un travail ethnographique, les auteurs retracent l'émergence et le développement de cette technologie. En outre, ils prêtent une attention particulière à l'expérience corporelle des utilisateurs du Fauteuil Tout Terrain, expérience qui amène à des «reconfigurations identitaires». Le second article de Howe et Silva focalise son attention sur le sport paralympique et y étudie plus particulièrement le processus de «cyborgyfication» associé à la technologisation des corps sportifs handicapés. Les auteurs montrent notamment comment la figure cyborg, si elle est susceptible de valoriser une poignée de sportifs, peut parallèlement discréditer les corps qui ne participent pas à ce processus de technologisation des corps. Ainsi, les sportifs qui n'utilisent pas de manière ostentatoire la technologie n'accèdent pas au statut de «supercrip » .

L'article de Semerjian est un essai original qui questionne le vieillissement au regard de l'usage des nouvelles technologies. À partir de la notion d'inspiration foucaldienne «bio-other», Tamar Semerjian met en balance la possible autonomie (positif) versus la surveillance (négatif) dont ces technologies peuvent être la source.

Dans leur article, Peter et Martin, à partir d'un croisement interdisciplinaire heuristique, privilégiant l'échange entre sciences sociales et sciences du vivant, font le point sur l'utilisation des technologies dans l'analyse des mouvements sportifs efficients voire de ses applications dans les processus d'entraînement. Pour illustrer leur propos, les auteurs privilégient le geste du service en tennis.

Le travail proposé par Suchet s'intéresse à l'émergence d'une technologie nouvelle au fondement des pratiques d'ascensionnisme dont l'histoire complète reste assez mal connue: le piton d'escalade. Ce travail qui croise les apports de la sociologie de l'innovation et de l'histoire des techniques, interroge bien au-delà de l'histoire des pratiques sportives, les conditions sociales, économiques et culturelles de l'adoption et de la diffusion d'un objet technique de montagne au cours de la première moitié du $\mathrm{xx}^{\mathrm{e}}$ siècle dans un contexte géopolitique spécifique. Enfin, Terret, Robène et Grosjean proposent une approche de l'histoire des techniques du canoë-kayak freestyle. Le but de cette étude qui croise les apports de l'histoire des techniques et de la technologie culturelle est de déterminer les mécanismes qui ont conduit à une évolution rapide des figures, incluant notamment les transformations du milieu technique, du matériel, des règlements, l'apport d'Internet, des images partagées et la construction de communautés pour lesquelles la comparaison de gestes techniques et de performance dynamise la concurrence et le défi. Les résultats montrent plus généralement que les figures réalisées par les pagayeurs sont de plus en plus aériennes, rapides, fluides et enchaînées.

Augmentation, autonomisation, incorporation, contrôle, règlements sont autant de notions que ces articles, en questionnant le sport et la technologie, nous invitent à investiguer au prisme de l'interdisciplinarité. 
Remerciements. Nous tenons à remercier Rémi Richard, MCF à l'UFR STAPS de Montpellier - Laboratoire Santé et Éducation, Situation de Handicap (Sant.E.Si.H.), EA4614, pour sa participation dans l'appel à contributions pour ce numéro spécial.

\section{Références}

Bret, P. (2004). Un bateleur de la science. Le «machinistephysicien » François Bienvenu et la diffusion de Franklin et Lavoisier, Annales historiques de la Révolution française, 338 , 95-127.

Castoriadis, C. (1995). Technique, Encyclopedia Universalis, 22, $123-129$.

Coppens, Y. (2009). Le Présent du passé. L'Actualité de l'histoire de l'Homme. Paris: Odile Jacobs.

Ellul, J. (1988). Le bluff technologique. Paris: Hachette.

Ehrenberg, A. (1991). Le culte de la performance. Paris: Calmann-Lévy.

Fèbvre, L. (1935). Réflexions sur l'histoire des techniques, Les Annales d'histoire économique et sociale, VII.

Habermas, J. (1973). La technique et la science comme idéologie. Paris : Gallimard.

Leibnitz, W. (1890). Discours touchant la méthode de la certitude et l'art d'inventer. In C.J. Gerhardt (Ed.), Die philosophischen schriften von Gottfried Wilhelm Leibnitz (pp. 174-183). Berlin: Wlidmannshe Buchhanlung.

Leroi-Gouhran, A. (1964). Le geste et la parole, tome I: Technique et langage. Paris: Albin Michel.

Loland, S. (2002). Technology in sport: Three ideal-typical views and their implications, European Journal of Sport Science, 2(1), 1-11.

Mauss, M. (1950). Sociologie et anthropologie. Paris: Presses universitaires de France.

Pociello, C. (1999). La science en mouvement. Etienne-Jules Marey et Georges Demeny (1870-1920). Paris: Presses universitaires de France.

Robène, L. (1998). L'homme à la conquête de l'air. 2 vol. Paris : L'Harmattan.

Robène, L. (2008). Sport et violences. L'éducation par le sport et les dispositifs de prévention de la violence, The International Journal on Violence and School, 6. Disponible sur: http:// www.ijvs.org/.

Robène, L. (2014). L'histoire des techniques et des technologies sportives : une matrice culturelle franco-française de l'histoire du sport? Science et motricité, 86, 93-104.

Robène, L., Léziart, Y. (2006). L'homme en mouvement. Histoire et anthropologie des techniques sportives. 2 vol. Paris : Chiron.

Robène, L., Bodin, D. (2014). Sport, Technique and Violence. The technical mode for generating sport violence, The International Journal of the History of Sport, 31-16, 2034-2058.

Vigarello, G. (1988). Techniques d'hier et d'aujourd'hui. Paris : Revue EPS - Robert Laffont.

Citation de l'article : Robène L (2017) Technologies et techniques des sports : le regard de l'histoire et des sciences humaines et sociales. Mov Sport Sci/Sci Mot, 97, 1-7 